\title{
Quintais agroflorestais em uma comunidade rural no vale do Rio Araguari, Amazônia Oriental
}

O objetivo deste trabalho foi caracterizar os quintais agroflorestais (QAF) do ponto de vista agroecológico, estrutural e florístico na comunidade de São Tomé município de Ferreira Gomes, Amapá. No trabalho de campo utilizou-se o método etnográfico com as técnicas de observação participante, entrevistas formais e informais. Além disso, foi realizado um inventário das plantas que compõem os quintais, levantando-se o número de espécies existentes, tipos de usos e estrutura dos QAF. As propriedades investigadas apresentaram tamanhos variados, bem como o número de espécies e espécimes. Animais domésticos são criados para principalmente complementar alimentação. Foram registrados nos quintais um total de 97 espécies vegetais, incluídas em 44 famílias e 80 gêneros. As famílias com maior número de espécies foram: Rutaceae, Fabaceae, Arecaceae, Malvaceae e Myrtaceae. Com relação às categorias de uso, a maioria das espécies é de uso múltiplo, com destaque para as de uso alimentar (58 espécies) e medicinal (31 espécies). As espécies mais frequentes de uso alimentar foram: Cocos nucifera L., Mangifera indica L., Theobroma grandiflorum (Willd. ex Spreng.) K. Schum, Musa cavendishii, Bactris gasipaes Kunth. E as espécies de uso medicinal mais frequentes foram: Carapa guianensis Aubl., Carica papaya L., Vernonia condensata Baker, Lippia alba (Mill.) N.E. Br. ex Britton \& P. Wilson, Ruta graveolens L. O manejo dos QAF é simples e de baixo custo, uma vez que envolve práticas tradicionais de cultivo de plantas e a principal finalidade dos quintais na comunidade São Tomé é de promover a complementação alimentar das unidades familiares e de venda de sua produção excedente na feira.

Palavras-chave: Sistema agroflorestal; Agricultura familiar; Segurança alimentar.

\section{Agroforestry backyards in a rural community in the Araguari River valley, Eastern Amazon}

\begin{abstract}
The objective of this work was to characterize homegardens (QAF) from an agroecological, structural and floristic point of view in the community of São Tomé municipality of Ferreira Gomes, Amapá. In the field work, ethnographic method was used with participant observation techniques, formal and informal interviews. In addition, an inventory of the plants that make up the homegardens was carried out, surveying the number of existing species, types of uses and structure of the QAF. The investigated properties presented varied sizes, as well as the number of species and specimens. Domestic animals are bred mainly to supplement food. A total of 97 plant species were registered in the backyards, included in 44 families and 80 genera. Botanical families with the largest number of species were: Rutaceae, Fabaceae, Arecaceae, Malvaceae and Myrtaceae. Regarding the categories of use, most species are for multiple uses, with emphasis on food (58 species) and medicinal (31 species). The most frequent species for food use were Cocos nucifera L., Mangifera indica L., Theobroma grandiflorum (Willd. Ex Spreng.) K. Schum, Musa cavendishii, Bactris gasipaes Kunth., And the most frequent species of medicinal use were: Carapa guianensis Aubl., Carica papaya L., Vernonia condensata Baker, Lippia alba (Mill.) N.E. Br. Ex Britton \& P. Wilson and Ruta graveolens L. The management of homegardens is simple and low cost, since it involves traditional practices of plant cultivation and the main purpose of backyards in the São Tomé community is to promote the food complementation of family units and to sell their surplus production at the fair.
\end{abstract}

Keywords: Agroforestry system; Family farming; Food security.

Topic: Agroecologia

Reviewed anonymously in the process of blind peer.
Received: 04/03/2021

Approved: 23/03/2021
João Ramos de Matos Filho (iD)

Universidade do Estado do Amapá, Brasil http://lattes.cnpq.br/5347907704912741

http://orcid.org/0000-0003-1331-8299

joaoramos ir@hotmail.com

Luiz Leno da Costa Moraes

Universidade do Estado do Amapá, Brasil http://lattes.cnpq.br/3573575901835911

http://orcid.org/0000-0002-8914-8719

lenomoraes2009@hotmail.com

João da Luz Freitas (iD)

Instituto de Estado do Amapá, Brasil

http://lattes.cnpq.br/7708025882561023

http://orcid.org/0000-0002-9751-9479

ifreitas.ap@bol.com.br
Francisco de Oliveira Cruz Junior

Instituto de Estado do Amapá, Brasil

http://lattes.cnpq.br/1485515135052878

http://orcid.org/0000-0003-3059-165X

francisco.florestal@bol.com.br

Adriano Castelo dos Santos

Instituto de Estado do Amapá, Brasil

http://lattes.cnpq.br/2674862974173349

http://orcid.org/0000-0002-8174-441X

adrianocasteloeng@gmail.com

\section{Referencing this:}

MATOS FILHO, J. R.; MORAES, L. L. C.; FREITAS, J. L.; CRUZ JUNIOR, F. O.; SANTOS, A. C.. Quintais agroflorestais em uma comunidade rural no vale do Rio Araguari, Amazônia Oriental. Revista Ibero Americana de Ciências Ambientais, v.12, n.3, p.47-62, 2021. DOI: 


\section{INTRODUÇÃO}

Os sistemas agroflorestais (SAF) são sistema de uso e manejo dos recursos naturais que integram consorciações de árvores e culturas agrícolas e/ou animais de forma científica, ecologicamente desejável, praticamente factível e socialmente aceitável pelo produtor rural, de modo que este obtenha os benefícios das interações ecológicas e econômicas resultantes (NAIR, 2011; ALVES et al., 2015; MARTINS et al., 2019).

Uma das modalidades de SAF são os quintais agroflorestais, definidos como sistemas de manejo tradicionais nos trópicos e considerados como sustentáveis ao longo dos anos, pois ofertam uma série de produtos e/ou serviços, diminuindo de forma considerável os gastos da família para obtê-los fora da propriedade. Os QAF caracterizam-se por uma imitação de ecossistemas naturais e assim requerem a utilização de baixos insumos, além de provocarem menos danos ao meio ambiente (DAS et al., 2005).

Os quintais agroflorestais são sistemas de manejo tradicionais nos trópicos e considerados como sistemas sustentáveis ao longo dos anos, pois oferecem uma série de produtos e/ou serviços, diminuindo de forma considerável os gastos da família para obtê-los fora da propriedade (CARVALHO et al., 2007).

Esse agroecossistema é um dos sistemas agroflorestais mais antigos e conhecidos nas regiões tropicais e é citado como sustentável por vários estudos (PEYRE et al., 2006). Uma das características dos quintais agroflorestais é sua complexidade, apresentando múltiplos estratos, incluindo muitas formas de vida, desde plantas trepadeiras, árvores, cultivos rasteiros e algumas vezes animais.

Na Amazônia, existem diversos sistemas agroflorestais em uso há muito tempo, desenvolvidos por comunidades indígenas, caboclas e ribeirinhas, principalmente para fins de sobrevivência, praticados por esses povos tradicionais, mas que precisam ser melhor descritos, pois podem constituir um conhecimento que corre o risco de ser perdido para sempre (SILVA, 2010).

Os QAF ofertam uma diversidade de produtos e benefícios ao longo do ano todo, alta diversidade de plantas, principalmente para uso humano, com arranjo similar às florestas naturais, eficiência no ciclo de nutrientes, redução do uso de insumos externos sintéticos, manejo baseado no conhecimento ecológico desenvolvido localmente e baixo impacto ao meio ambiente (CARNEIRO et al., 2013; FREITAS et al., 2015; GOMES et al., 2018). Os quintais agroflorestais desempenham importantes papéis sociais, como a produção de alimentos para consumo familiar, principal motivação para a adoção desses agroecossistemas. Esses espaços fazem parte do cotidiano e da dinâmica de muitas famílias da Amazônia, contribuindo no fortalecimento das relações interpessoais, manutenção de tradições e costumes que são fortemente atrelados ao uso da agrobiodiversidade (RAYOL et al., 2019).

Nesse contexto, a comunidade de São Tomé, que terá sua área alagada devido à construção das hidrelétricas Cachoeira Caldeirão e Ferreira Gomes Energia perderá área produtiva, ocasionando perda da biodiversidade, perdas de alimentação e renda daquela comunidade que depende de seus quintais agroflorestais para sua subsistência. Além disso, corre o risco que se perca todo o conhecimento tradicional adquirido durante muitos anos dos seus quintais agroflorestais.

Diante desse fato, torna-se imprescindível a realização do estudo de caracterização dos quintais 
agroflorestais da comunidade de São Tomé para registrar esses conhecimentos e evitar assim a perda perene. Vale ressaltar que este estudo também poderá auxiliar a população daquela comunidade a implantar novamente seus quintais agroflorestais na nova área para onde serão realocados. Assim, a pergunta norteadora deste trabalho é: qual a composição florística dos quintais agroflorestais da comunidade de São Tomé, no município de Ferreira Gomes, bem como sua organização estrutural?

Desta forma, este estudo objetivou caracterizar os quintais agroflorestais do ponto de vista agroecológico, estrutural e florístico em uma comunidade rural no Vale do rio Araguari, município de Ferreira Gomes, Estado do Amapá.

\section{MATERIAIS E MÉTODOS}

\section{Localização da área de estudo}

A pesquisa foi realizada na Comunidade de São Tomé, localizada dentro da Reserva Particular do Patrimônio Natural (RPPN) Seringal Triunfo, no município de Ferreira Gomes (Figura 1), na mesorregião sul do estado do Amapá, a 132 km, da capital Macapá. Ferreira Gomes. A comunidade limita-se ao norte com o município de Pracuúba, ao sul com Macapá, ao leste com Tartugalzinho e Cutias do Araguari e a oeste com Serra do Navio, Pedra Branca do Amapari e Porto Grande.

Ferreira Gomes possui uma área de 5.046,696 km², o que representa 3,53\% do território do Estado do Amapá e 0,059\% do território brasileiro. Abrange os distritos Ferreira Gomes e Paredão, além das comunidades Igarapé, Roberta, Campinho, Igarapé da Onça, Zebulândia, Onório, Igarapé do Palha e Triunfo do Araguari. Sua população é de 5.802 habitantes, densidade demográfica 1,14 hab/km² e grau de urbanização 72,09\% (FERREIRA GOMES, 2013).

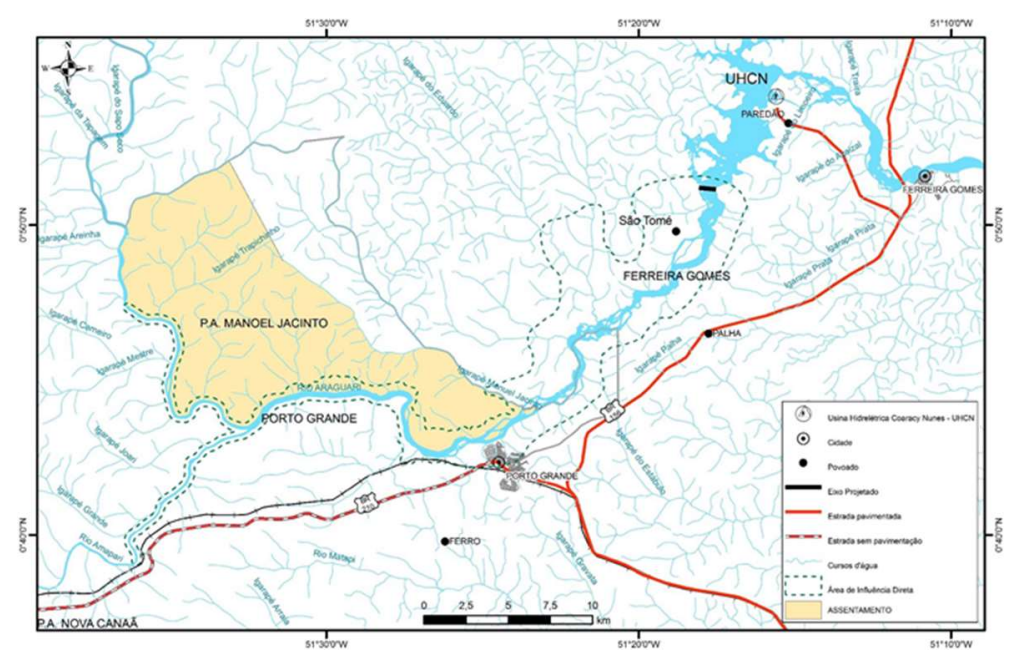

Figura 1: Localização da comunidade São Tomé, Ferreira Gomes, Amapá. Fonte: Ecotumucumaque (2010).

A região de Ferreira Gomes possui, de acordo com a classificação Köppen, clima do tipo Am, onde o "A" indica um clima tropical e o " $\mathrm{m}$ " aponta para um clima de monção, com precipitações totais anuais médias maiores do que $1.500 \mathrm{~mm}$ e precipitações nos meses mais secos menores do que $60 \mathrm{~mm}$. Esta classificação microclimática caracteriza a área como de clima tropical úmido de monção, com precipitação excessiva durante alguns meses, o que compensa a ocorrência de um ou dois meses com precipitações 
inferiores a $60 \mathrm{~mm}$, condição visualizada na maior parte da Amazônia. A estação seca é de curta duração, apresentando uma quantidade pluviométrica no mês menos chuvoso igual ou superior a $60 \mathrm{~mm}$ (ECOTUMUCUMAQUE, 2010).

Ferreira Gomes insere-se na região da Bacia do Araguari, composta pelo rio homônimo e pela Foz do Araguari. Destaca-se que, essa bacia, dada sua magnitude, com aproximadamente $42 \mathrm{mil} \mathrm{km}^{2}$ de área total e vazão média de $1.200 \mathrm{~m}^{3} / \mathrm{s}$ apresenta um excepcional potencial energético (AMAPÁ, 2009), que tem sido motivo de estudos e de investimentos na construção de aproveitamentos hidrelétricos, podendo-se citar o caso recente da construção da UHE Ferreira Gomes e da UHE Cachoeira Caldeirão no município, além da UHE Coaracy Nunes em funcionamento desde 1976. O Rio Araguari é considerado o principal rio do Amapá. Com cerca de $617 \mathrm{~km}$ de comprimento e índice de drenagem da ordem de 0,955/km, configura-se como o maior rio do Estado.

Os latossolos predominam na região onde se insere o município do Ferreira Gomes, com esparsas variações localmente relacionadas com argissolos. De acordo com o Estudo de Impacto Ambiental do AHE Ferreira Gomes, estes tipos de solos encontram-se em fase senil, evoluídos, com baixa fertilidade natural, pouca diferenciação no teor de argila em profundidade e estrutura tipicamente granular. Em geral, são solos fortemente ácidos e encontram-se distribuídos, sobretudo, por antigas superfícies de erosão, pedimentos ou terraços fluviais, normalmente em relevo plano e suave, embora possam ocorrer também em áreas mais acidentadas.

De acordo com o levantamento botânico realizado para o diagnóstico ambiental do AHE Ferreira Gomes (ECOTUMUCUMAQUE, 2010), destacam-se na área do município de Ferreira Gomes quatro fitofisionomias principais, a saber: Floresta Ombrófila Densa, Cerrado, Floresta de Várzea e Floresta Secundária (capoeira).

\section{Coleta de dados}

Em julho de 2014, foi realizada uma reunião com a comunidade, intermediada pela presidente da Associação dos Agroextrativistas da Comunidade de São Tomé do Rio Araguari (ASPROARI). A metodologia adotada foi definida após viagem para pesquisa exploratória e solicitação de anuência dos moradores para a realização da pesquisa. De início, foi realizado um levantamento das unidades domiciliares existentes na área estudada para conhecimento do universo da pesquisa, durante o qual foram catalogadas um total de 21 propriedades onde havia presença de residência. A partir deste levantamento, decidiu-se pela coleta de dados em todos os domicílios, ou seja, a realização de um censo. Vale ressaltar que havia 36 propriedades inscritas na ASPROARI.

Os procedimentos metodológicos adotados para o levantamento de dados sobre os QAF e o estudo etnobotânico foram determinados como sugere Amorozo (1996), Gil (1999), Silva (2002; 2010) e CoelhoFerreira (2009). Utilizou-se as entrevistas estruturadas, as entrevistas informais e a observação participante. As entrevistas foram realizadas no ambiente dos próprios entrevistados e ocorreram, preferencialmente, com os responsáveis diretamente pelo grupo familiar, independente de gênero. 
Além disso, foi realizado um inventário das plantas nos QAF dos produtores rurais da comunidade São Tomé, levantando-se o número de espécies existentes nos mesmos, bem como seus usos para a família. Em cada quintal um representante da família, de preferência a pessoa que cuidava do mesmo, fez companhia e servia de guia para assim identificar as espécies pelos seus nomes vernaculares e seus principais atributos de utilidade relacionados a categorias de uso.

Para a coleta das espécies foi utilizado a metodologia de acordo com Coelho-Ferreira (2009) e Silva (2010), ou seja, as amostras coletadas foram processadas em campo e cada uma recebeu um número de coleta, marcada em etiqueta previamente preparada, que foi amarada a mesma, contendo seu nome vernacular. Concomitantemente foi realizado o registro fotográfico das espécies para auxiliar na identificação botânica das mesmas. Para a identificação das famílias utilizou-se o sistema de classificação Angiosperm Phylogeny Group III (APG, 2009) e com comparação no Herbário Amapaense (HAMAB). Para conferência da grafia e sinonímia foi consultado o banco de dados do The Plant List e Missouri Botanical Garden.

Os dados coletados e registrados nas cadernetas de campo e nos formulários foram organizados e sistematizados em fichas por domicílio rural entrevistado. Em seguida, foram tabulados e sintetizados através do programa Microsoft 2010 para a formação de um banco de dados.

\section{RESULTADOS}

Foram realizadas 13 entrevistas em propriedades rurais da comunidade São Tomé, sendo que seis estão localizadas às margens do Rio Araguari e sete em terra firme. A faixa etária da população amostrada existente na área de estudo foi de 28 a 68 anos. Um aspecto observado foi o baixo nível de instrução entre os moradores da comunidade, aqueles que estudaram até a 4a do ensino fundamental representam o maior número, 70\% dos informantes.

\section{Aspectos gerais dos Quintais agroflorestais}

A área das propriedades investigadas variou de 2,2 ha até 100 ha, sendo que o tamanho mais frequente dos mesmos foi de 50 ha, o que representa $46,15 \%$ das propriedades amostradas (Tabela 1 ).

Tabela 1: Área física das propriedades na Comunidade São Tomé, Ferreira Gomes - AP.

\begin{tabular}{lllll}
\hline $\begin{array}{l}\text { Área (largura } \\
\text { comprimento) }\end{array}$ & $\begin{array}{l}\text { Área em } \\
\mathbf{m}^{\mathbf{2}}\end{array}$ & $\begin{array}{l}\text { Área em } \\
\text { hectares }\end{array}$ & $\begin{array}{l}\text { Número de } \\
\text { propriedades }\end{array}$ & $\begin{array}{l}\text { Frequência } \\
\text { relativa (em \%) }\end{array}$ \\
\hline $100 \times 200$ & 20000 & 2 & 1 & 7,69 \\
$100 \times 900$ & 90000 & 9 & 1 & 7,69 \\
$260 \times 800$ & 208000 & 20,8 & 2 & 15,38 \\
$500 \times 1000$ & 500000 & 50 & 6 & 46,15 \\
$1000 \times 1000$ & 1000000 & 100 & 3 & 23,08 \\
\hline Total & & & $\mathbf{1 3}$ & $\mathbf{1 0 0 , 0 0}$ \\
\hline
\end{tabular}

Em todos os quintais a força de trabalho utilizada é a humana, em 23,08\% deles é usada também a força animal e em nenhum é utilizada força mecânica. A maioria dos agricultores estudados ( $70 \%)$ não utiliza assistência técnica para as atividades agrícolas relacionadas aos quintais e 30,77\% utilizam ocasionalmente e nenhum com regularidade. Todos os entrevistados que utilizam ocasionalmente a assistência técnica tem como finalidade a melhoria da produtividade. 
Os cuidados dispensados ao quintal, como o plantio de espécies, tratos culturais como capina, limpeza de áreas e rega são realizados plenamente por membros familiares da unidade domiciliar, e a participação do homem e da mulher nesse manejo representa 70\%.

\section{Criação de animais nos QAF}

Na comunidade São Tomé essa realidade também se faz presente e 61,54\% dos quintais estudados, animais são criados com a finalidade principal de consumo e em alguns poucos casos também para comercialização com o intuito de geração de renda. Os animais que são criados nos quintais são: galinhas (61,54\% dos quintais), patos (23,08\%), porcos (23,08\%), peru $(7,69 \%)$ e bois $(15,38 \%)$ (Tabela 2$)$.

Tabela 2: Dados sobre os animais criados nos quintais da Comunidade São Tomé, Ferreira Gomes-AP.

\begin{tabular}{llll}
\hline \multirow{2}{*}{ Animal } & Quintais & Frequência relativa & Total de indivíduos \\
\cline { 2 - 4 } & Presença de animais & 61,54 & 195 \\
\hline Galinha & 8 & 23,08 & 33 \\
Pato & 3 & 23,08 & 16 \\
Porco & 3 & 7,69 & 5 \\
Peru & 1 & 15,38 & 8 \\
Boi & 2 & & $\mathbf{2 5 7}$ \\
\hline Total & & & \\
\hline
\end{tabular}

\section{Arranjo estrutural dos QAF}

Baseado na classificação em um autor que pesquisou em 1992, os quintais apresentam um arranjo estrutural de quatro zonas distintas, que são: 1을 Estrato (até $1 \mathrm{~m}$ de altura): composto por espécies herbáceas, sendo as preferenciais os cultivos medicinais e alimentícios e algumas ornamentais como Ananas comosus (L.) Merr. (Abacaxi) e Chenopodium ambrosioides L. (mastruz); 2o estrato (de $1 \mathrm{~m}$ até $2 \mathrm{~m}$ ): composto por arbustos jovens, de hortaliças e espécies medicinais, como Lippia alba (Mill.) N.E. Br. (cidreira) e Capsicum frutescens L. (pimenta malagueta); 3o Estrato (de $2 \mathrm{~m}$ até $5 \mathrm{~m}$ ): composto por espécies arbustivas e árvores pequenas, como Anacardium occidentale L. (cajueiro), Annona muricata L. (gravioleira) e Musa cavendishii Lamb. ex Paxton (bananeira); e 4을 Estrato (de 5 até 30m): composto por espécies madeireiras e frutíferas, como Cocos nucifera L. (coqueiro), Euterpe oleracea Mart. (açaizeiro) e Mangifera indica L. (mangueira).

\section{Espécies botânicas registradas nos QAF}

Nos quintais agroflorestais da comunidade São Tomé foram registradas 97 espécies vegetais pertencentes a 43 famílias e 80 gêneros.

O número de espécies registradas por quintal variou entre 10 e 41 espécies, com média de 23,85 por quintal. Os QAF-8 (41 espécies), QAF-2 (35 espécies), QAF-7 (35 espécies) foram considerados os mais exitosos, pois apresentaram a maior riqueza de espécies e em contrapartida, os quintais QAF-5 (10 espécies) e QAF-10 (14 espécies) foram os que apresentaram o menor número (Tabela 3).

Tabela 3: Riqueza de espécies vegetais nos quintais agroflorestais da Comunidade São Tomé, com as frequências absolutas (FA) e relativas (FR-\%).

\begin{tabular}{lll}
\hline QAF & FA & FR(\%) \\
\hline 1 & 18 & 18,5
\end{tabular}




$\begin{array}{ll}35 & 36,08 \\ 17 & 17,53 \\ 24 & 24,74 \\ 10 & 10,31 \\ 20 & 20,62 \\ 35 & 36,08 \\ 41 & 42,27 \\ 26 & 26,80 \\ 14 & 14,43 \\ 17 & 17,53 \\ 22 & 22,68 \\ 31 & 31,96\end{array}$

No Quadro 1, as espécies botânicas são apresentadas com as suas respectivas nomenclaturas científicas.

Quadro 1: Riqueza de espécies dos quintais agroflorestais da comunidade São Tomé, Ferreira Gomes - AP.

\begin{tabular}{|c|c|}
\hline Família / Nome Científico & Nome Vernacular \\
\hline \multicolumn{2}{|l|}{ ANACARDIACEAE } \\
\hline Anacardium occidentale L. & Cajueiro \\
\hline Mangifera indica $\mathrm{L}$. & Mangueira \\
\hline Spondias mombin L. & Taperebazeiro \\
\hline \multicolumn{2}{|l|}{ ANNONACEAE } \\
\hline Annona squamosa $\mathrm{L}$. & Ata \\
\hline Rollinia mucosa (Jacq.) Baill. & Biribazeiro \\
\hline Annona muricata L. & Gravioleira \\
\hline \multicolumn{2}{|l|}{ ARACEAE } \\
\hline Caladium lindenii (André) Madison & Brasileirinho \\
\hline \multicolumn{2}{|l|}{ ARECACEAE } \\
\hline Euterpe oleracea Mart. & Açaizeiro \\
\hline Oenocarpus bacaba Mart. & Bacabeira \\
\hline Cocos nucifera $\mathrm{L}$ & Coqueiro \\
\hline Oenocarpus bataua Mart. & Patauá \\
\hline Bactris gasipaes Kunth & Pupunheira \\
\hline \multicolumn{2}{|l|}{ ASTERACEAE } \\
\hline Spilanthes oleracea $\mathrm{L}$. & Jambú \\
\hline Vernonia condensata Baker & Boldo \\
\hline Galinsoga parviflora Cav. & Picão \\
\hline \multicolumn{2}{|l|}{ BIGNONIACEAE } \\
\hline Crescentia cujete L. & Cuieira \\
\hline Arrabidaea chica (Humb. \& Bonpl.) B.Verl. & Pariri \\
\hline \multicolumn{2}{|l|}{ BIXACEAE } \\
\hline Bixa orellana $\mathrm{L}$. & Urucunzeiro \\
\hline \multicolumn{2}{|l|}{ BROMELIACEAE } \\
\hline Ananas comosus (L.) Merr. & Abacaxizeiro \\
\hline \multicolumn{2}{|l|}{ CARICACEAE } \\
\hline Carica papaya L. & Mamoeiro \\
\hline \multicolumn{2}{|l|}{ CARYOCARACEAE } \\
\hline Caryocar villosum (Aubl.) Pers. & Piquiazeiro \\
\hline \multicolumn{2}{|l|}{ CHENOPODIACEAE } \\
\hline Chenopodium ambrosioides L. & Mastruz \\
\hline \multicolumn{2}{|l|}{ CONVOLVULACEAE } \\
\hline Ipomoea batatas (L.) Lam. & Batata doce \\
\hline \multicolumn{2}{|l|}{ CRASSULACEAE } \\
\hline Bryophyllum calycinum Salisb. & Pirarucú \\
\hline \multicolumn{2}{|l|}{ CUCURBITACEAE } \\
\hline Cucurbita pepo L. & Abóbora/Jerimum \\
\hline Cucumis anguria $\mathrm{L}$. & Maxixe \\
\hline \multicolumn{2}{|l|}{ CUPRESSACEAE } \\
\hline Cupressus Iusitanica Mill. & Cedrinho \\
\hline \multicolumn{2}{|l|}{ DIOSCOREACEAE } \\
\hline Dioscorea sp. & Cará \\
\hline \multicolumn{2}{|l|}{ EUPHORBIACEAE } \\
\hline Manihot esculenta Crantz & Mandioca/Macaxeira \\
\hline
\end{tabular}




\begin{tabular}{|c|c|}
\hline Hevea brasiliensis Müll.Arg. & Seringueira \\
\hline Pedilanthus tithymaloides (L.) Poit. & Coramina \\
\hline \multicolumn{2}{|l|}{ FABACEAE } \\
\hline Hymenolobium excelsum Ducke & Angelim \\
\hline Copaifera sp. & Copaibeira \\
\hline Inga edulis Mart. & Ingazeiro \\
\hline Hymenaea courbaril L. & Jatobá \\
\hline Pentaclethra macroloba (Willd.) Kuntze & Pracaxizeiro \\
\hline Dalbergia monetaria L. f. & Verônica \\
\hline \multicolumn{2}{|l|}{ ICACINACEAE } \\
\hline Poraqueiba paraensis Ducke & Marim \\
\hline \multicolumn{2}{|l|}{ LAMIACEAE } \\
\hline Ocimum minimum L. & Manjericão \\
\hline Aeollanthus suaveolens Mart. ex Spreng. & Catinga-de-mulata \\
\hline Plectranthus amboinicus (Lour.) Spreng. & Hortelã \\
\hline Mentha pulegium $\mathrm{L}$. & Hortelanzinho \\
\hline \multicolumn{2}{|l|}{ LAURACEAE } \\
\hline Persea americana Mill. & Abacateiro \\
\hline Cinnamomum zeylanicum Blume & Caneleira \\
\hline \multicolumn{2}{|l|}{ XANTHORRHOCACEAE } \\
\hline Aloe vera (L.) Burm. f. & Babosa \\
\hline \multicolumn{2}{|l|}{ LYTHRACEAE } \\
\hline Punica granatum L. & Romanzeiro \\
\hline \multicolumn{2}{|l|}{ MALPIGHIACEAE } \\
\hline Malpighia punicifolia L. & Aceroleira \\
\hline Byrsonima crassifolia (L.) Kunth & Muricizeiro \\
\hline \multicolumn{2}{|l|}{ MALVACEAE } \\
\hline Gossypium arboreum L. & Algodoeiro \\
\hline Herrania mariae (Mart.) Decne. ex Goudot & Cacau do mato \\
\hline Hibiscus esculentus L. & Quiabo \\
\hline Hibiscus sabdariffa $\mathrm{L}$. & Vinagreira \\
\hline Theobroma cacao L. & Cacaueiro \\
\hline Theobroma grandiflorum (Willd. ex Spreng.) K. Schum. & Cupuzeiro \\
\hline \multicolumn{2}{|l|}{ MELIACEAE } \\
\hline Carapa guianensis Aubl. & Andirobeira \\
\hline Cedrela odorata $\mathrm{L}$. & Cedro \\
\hline Swietenia macrophylla King & Mogno \\
\hline \multicolumn{2}{|l|}{ MORACEAE } \\
\hline Artocarpus heterophyllus Lam. & Jaca da bahia \\
\hline Artocarpus altilis (Parkinson) Fosberg & Fruta pão \\
\hline \multicolumn{2}{|l|}{ MUSACEAE } \\
\hline Musa cavendishii Lamb. ex Paxton & Bananeira \\
\hline \multicolumn{2}{|l|}{ MYRTACEAE } \\
\hline Syzygium cumini (L.) Skeels & Ameixeira \\
\hline Eucalyptus sp. & Eucalipto \\
\hline Psidium acutangulum DC. & Goiaba Araçá \\
\hline Psidium guajava $\mathrm{L}$. & Goiabeira \\
\hline Eugenia malaccensis $\mathrm{L}$. & Jambeiro \\
\hline \multicolumn{2}{|l|}{ OXALIDACEAE } \\
\hline Averrhoa carambola $\mathrm{L}$. & Caramboleira \\
\hline Averrhoa bilimbi L. & Limão Caiena \\
\hline \multicolumn{2}{|l|}{ PEDALIACEAE } \\
\hline Sesamum indicum $\mathrm{L}$. & Gergilim \\
\hline \multicolumn{2}{|l|}{ PHYTOLACCACEAE } \\
\hline Petiveria alliacea $\mathrm{L}$. & Mucuracaá \\
\hline \multicolumn{2}{|l|}{ PIPERACEAE } \\
\hline Piper nigrum L. & Pimenta do reino \\
\hline Piper callosum Ruiz \& Pav. & Elixir paregórico \\
\hline POACEAE & \\
\hline Saccharum officinarum L. & Cana-de-açúcar \\
\hline Cymbopogon citratus (DC.) Stapf & Capim marinho/Capim santo \\
\hline PORTULACACEAE & \\
\hline Portulaca pilosa $\mathrm{L}$. & Amor crescido \\
\hline RUBIACEAE & \\
\hline Coffea arabica L. & Cafeeiro \\
\hline
\end{tabular}




\begin{tabular}{|c|c|}
\hline Genipa americana L. & Jenipapeiro \\
\hline Morinda citrifolia $\mathrm{L}$. & Noni \\
\hline Uncaria tomentosa (Willd. ex Roem. \& Schult.) DC. & Unha-de-gato \\
\hline \multicolumn{2}{|l|}{ RUTACEAE } \\
\hline Citrus sinensis (L.) Osbeck & Laranjeira \\
\hline Citrus aurantium L. & Laranjeira da Terra \\
\hline Citrus aurantifolia (Christm.) Swingle & Lima \\
\hline Citrus aurantifolia (Christm.) Swingle & Limão Galego \\
\hline Citrus sp. & Limão Cidra \\
\hline Citrus limonia (L.) Osbeck. & Limoeiro \\
\hline Citrus nobilis Lour. & Tangerineira \\
\hline Ruta graveolens L. & Arruda \\
\hline \multicolumn{2}{|l|}{ SALICACEAE } \\
\hline Banara guianensis Aubl. & Andorinheira \\
\hline \multicolumn{2}{|l|}{ SAPOTACEAE } \\
\hline Pouteria caimito (Ruiz \& Pav.) Radlk. & Abiuzeiro \\
\hline Manilkara huberi (Ducke) Standl. & Maçaranduba \\
\hline Manilkara zapota (L.) P. Royen & Sapotizeiro \\
\hline \multicolumn{2}{|l|}{ SOLANACEAE } \\
\hline Capsicum frutescens L. & Pimenta malagueta \\
\hline Capsicum annuum L. & Pimentão \\
\hline Capsicum sp. & Pimentinha \\
\hline Solanum ovigerum L. & Jurú \\
\hline \multicolumn{2}{|l|}{ STYRACACEAE } \\
\hline Styrax argenteus C. Presl & Estoraque \\
\hline \multicolumn{2}{|l|}{ VERBENACEAE } \\
\hline Lippia alba (Mill.) N.E. Br. ex Britton \& P. Wilson & Erva Cidreira/cidreira \\
\hline \multicolumn{2}{|l|}{ ZINGIBERACEAE } \\
\hline Costus spicatus (Jacq.) Sw. & Canaficha \\
\hline
\end{tabular}

Das 43 famílias botânicas registradas, as que mais se destacaram pelo maior número de espécies foram: Rutaceae (oito espécies), Fabaceae e Malvaceae (6 espécies cada), Arecaceae e Myrtaceae (cinco espécies cada), Lamiaceae, Rubiaceae e Solanaceae (quatro espécies cada). As outras 35 famílias tiveram frequência igual ou menor que três espécies (Quadro 1).

\section{Disponibilidade das espécies nos QAF}

As espécies registradas nos quintais, $65,85 \%$ são obtidas de cultivos, $23,58 \%$ são as espontâneas e 10,57\% das espécies são obtidas de ambas as formas. Como exemplo de espécies cultivadas mais importantes têm-se Cocos nucifera (coqueiro), Mangifera indica (mangueira), Theobroma grandiflorum (cupuaçuzeiro), Musa cavendishii (bananeira), Citrus limonia (limoeiro), Citrus sinensis (laranjeira), Gravioleira (Annona muricata L.), Tangerineira (Citrus nobilis).

\section{Categorias de uso das espécies nos QAF}

As espécies encontradas nos QAF foram registradas em três etnocategorias principais: alimentar, medicinal e uso madeireiro. Sendo que, algumas espécies utilizadas para o artesanato, ornamental e arborização apareceram em menor número.

Foram registradas 58 espécies usadas pela comunidade na alimentação ou complemento desta (e também para a comercialização). As espécies que obtiveram maior destaque por ocorrência foram: Cocos nucifera (coqueiro), Mangifera indica (mangueira), Theobroma grandiflorum (cupuaçuzeiro), Musa cavendishii (bananeira), Bactris gasipaes (pupunheira), Euterpe oleracea (açaizeiro), Psidium guajava 
(goiabeira), Citrus limonia (limoeiro), Syzygium cumini (ameixeira), Citrus sinensis (laranjeira), Annona muricata (gravioleira), Inga edulis (ingazeiro) e Citrus nobilis (tangerineira).

Na Tabela 4 é apresentada a relação das espécies alimentícias usadas pela comunidade São Tomé, com nome científico, nome vernacular, frequência absoluta (FA) e a frequência relativa (\%).

Tabela 4: Importância relativa das espécies alimentícias por ocorrência nos quintais agroflorestais da comunidade São Tomé-AP.

\begin{tabular}{|c|c|c|c|c|}
\hline Nome Científico & Nome Vernacular & Família & FA & $\%$ \\
\hline Cocos nucifera $\mathrm{L}$. & Coqueiro & Arecaceae & 12 & 92,31 \\
\hline Mangifera indica $\mathrm{L}$. & Mangueira & Anacardiaceae & 12 & 92,31 \\
\hline Theobroma grandiflorum (Willd. ex Spreng.) K. Schum. & Cupuzeiro & Malvaceae & 11 & 84,62 \\
\hline Musa cavendishii Lamb. ex Paxton & Bananeira & Musaceae & 10 & 76,92 \\
\hline Bactris gasipaes Kunth & Pupunheira & Arecaceae & 10 & 76,92 \\
\hline Euterpe oleracea Mart. & Açaizeiro & Arecaceae & 9 & 69,23 \\
\hline Psidium guajava $\mathrm{L}$. & Goiabeira & Myrtaceae & 9 & 69,23 \\
\hline Citrus limonia (L.) Osbeck & Limoeiro & Rutaceae & 9 & 69,23 \\
\hline Syzygium cumini (L.) Skeels & Ameixeira & Myrtaceae & 8 & 61,54 \\
\hline Citrus sinensis (L.) Osbeck & Laranjeira & Rutaceae & 8 & 61,54 \\
\hline Annona muricata $\mathrm{L}$. & Gravioleira & Annonaceae & 7 & 53,85 \\
\hline Inga edulis Mart. & Ingazeiro & Fabaceae & 7 & 53,85 \\
\hline Citrus nobilis Lour. & Tangerineira & Rutaceae & 7 & 53,85 \\
\hline Malpighia punicifolia L. & Aceroleira & Malpighiaceae & 6 & 46,15 \\
\hline Anacardium occidentale L. & Cajueiro & Anacardiaceae & 6 & 46,15 \\
\hline Artocarpus heterophyllus Lam. & Jaca da bahia & Moraceae & 6 & 46,15 \\
\hline Persea americana Mill. & Abacateiro & Lauraceae & 5 & 38,46 \\
\hline Eugenia malaccensis L. & Jambeiro & Myrtaceae & 5 & 38,46 \\
\hline Citrus aurantifolia (Christm.) Swingle & Lima & Rutaceae & 5 & 38,46 \\
\hline Carica papaya L. & Mamoeiro & Caricaceae & 5 & 38,46 \\
\hline Byrsonima crassifolia (L.) Kunth & Murici & Malpighiaceae & 5 & 38,46 \\
\hline Ananas comosus (L.) Merr. & Abacaxizeiro & Bromeliaceae & 4 & 30,77 \\
\hline Oenocarpus bacaba Mart. & Bacabeira & Arecaceae & 4 & 30,77 \\
\hline Rollinia mucosa (Jacq.) Baill. & Biribazeiro & Annonaceae & 4 & 30,77 \\
\hline Theobroma cacao L. & Cacaueiro & Malvaceae & 4 & 30,77 \\
\hline Saccharum officinarum L. & Cana-de-açúcar & Poaceae & 4 & 30,77 \\
\hline Manihot esculenta Crantz & Mandioca/Macaxeira & Euphorbiaceae & 4 & 30,77 \\
\hline Spondias mombin L. & Taperebazeiro & Anacardiaceae & 4 & 30,77 \\
\hline Bixa orellana $\mathrm{L}$. & Urucunzeiro & Bixaceae & 4 & 30,77 \\
\hline Averrhoa carambola L. & Caramboleira & Oxalidaceae & 3 & 23,08 \\
\hline Solanum ovigerum L. & Jurú & Solanaceae & 3 & 23,08 \\
\hline Piper nigrum L. & Pimenta do reino & Piperaceae & 3 & 23,08 \\
\hline Caryocar villosum (Aubl.) Pers. & Piquiazeiro & Caryocaraceae & 3 & 23,08 \\
\hline Pouteria caimito (Ruiz \& Pav.) Radlk. & Abiuzeiro & Sapotaceae & 2 & 15,38 \\
\hline Annona squamosa $\mathrm{L}$. & Ata & Annonaceae & 2 & 15,38 \\
\hline Ipomoea batatas (L.) Lam. & Batata doce & Convolvulaceae & 2 & 15,38 \\
\hline Coffea arabica $\mathrm{L}$. & Cafeeiro & Rubiaceae & 2 & 15,38 \\
\hline Cinnamomum zeylanicum Blume & Caneleira & Lauraceae & 2 & 15,38 \\
\hline Artocarpus altilis (Parkinson) Fosberg & Fruta pão & Moraceae & 2 & 15,38 \\
\hline Psidium acutangulum DC. & Goiaba Araçá & Myrtaceae & 2 & 15,38 \\
\hline Citrus aurantifolia (Christm.) Swingle & Limão Galego & Rutaceae & 2 & 15,38 \\
\hline Oenocarpus bataua Mart. & Patauá & Arecaceae & 2 & 15,38 \\
\hline Capsicum frutescens $\mathrm{L}$. & Pimenta malagueta & Solanaceae & 2 & 15,38 \\
\hline Cucurbita pepo L. & Abóbora/Jerimum & Cucurbitaceae & 1 & 7,69 \\
\hline Herrania mariae (Mart.) Decne. ex Goudot & Cacau do mato & Malvaceae & 1 & 7,69 \\
\hline Dioscorea sp. & Cará & Dioscoreaceae & 1 & 7,69 \\
\hline Spilanthes oleracea $\mathrm{L}$. & Jambú & Asteraceae & 1 & 7,69 \\
\hline Genipa americana $\mathrm{L}$. & Jenipapeiro & Rubiaceae & 1 & 7,69 \\
\hline Citrus aurantium L. & Laranjeira da Terra & Rutaceae & 1 & 7,69 \\
\hline Averrhoa bilimbi L. & Limão Caiena & Oxalidaceae & 1 & 7,69 \\
\hline Citrus sp & Limão Cidra & Rutaceae & 1 & 7,69 \\
\hline Poraqueiba paraensis Ducke & Marim & Icacinaceae & 1 & 7,69 \\
\hline Cucumis anguria $\mathrm{L}$. & Maxixe & Cucurbitaceae & 1 & 7,69 \\
\hline Capsicum annuum L. & Pimentão & Solanaceae & 1 & 7,69 \\
\hline Capsicum sp. & Pimentinha & Solanaceae & 1 & 7,69 \\
\hline Hibiscus esculentus L. & Quiabo & Malvaceae & 1 & 7,69 \\
\hline
\end{tabular}


Punica granatum L.

Romanzeiro

Lythraceae

17,69

Manilkara zapota (L.) P. Royen

Sapotizeiro

Sapotaceae

17,69

As espécies com maior ocorrência nos quintais são de uso múltiplo, ou seja, além do uso medicinal, também são usadas de outras formas, como alimentar, arborização, madeireira ou ornamental. Vinte e uma espécies apresentam um percentual de ocorrência entre 7,69\% a 15,38\%, sendo que 19 destas aparecem somente em um quintal.

Na Tabela 5 é apresentada a relação das espécies medicinais usadas pela comunidade, com nome científico, nome vernacular, frequência absoluta (FA) e a relativa (\%). Foram registradas nos quintais agroflorestais 31 espécies utilizadas como medicinais na comunidade, nenhuma foi encontrada em todos os quintais, pois $\sim 85 \%$ dos produtores faziam uso de plantas medicinais.

Tabela 5: Importância relativa das espécies medicinais por ocorrência nos quintais da comunidade São Tomé-AP (2014).

\begin{tabular}{|c|c|c|c|c|}
\hline Nome Científico & Nome Vernacular & Família & FA & $\%$ \\
\hline Carapa guianensis Aubl. & Andirobeira & Meliaceae & 6 & 46,15 \\
\hline Carica papaya L. & Mamoeiro & Caricaceae & 5 & 38,46 \\
\hline Vernonia condensata Baker & Boldo & Asteraceae & 4 & 30,77 \\
\hline Lippia alba (Mill.) N.E. Br. ex Britton \& P. Wilson & Erva Cidreira/cidreira & Verbenaceae & 4 & 30,77 \\
\hline Ruta graveolens $\mathrm{L}$. & Arruda & Rutaceae & 3 & 23,08 \\
\hline Piper callosum Ruiz \& Pav. & Elixir paregórico & Piperaceae & 3 & 23,08 \\
\hline Plectranthus amboinicus (Lour.) Spreng. & Hortelã & Lamiaceae & 3 & 23,08 \\
\hline Morinda citrifolia $\mathrm{L}$. & Noni & Rubiaceae & 3 & 23,08 \\
\hline Arrabidaea chica (Humb. \& Bonpl.) B.Verl. & Pariri & Bignoniaceae & 3 & 23,08 \\
\hline Uncaria tomentosa (Willd. ex Roem. \& Schult.) DC. & Unha-de-gato & Rubiaceae & 3 & 23,08 \\
\hline Ocimum minimum $\mathrm{L}$. & Manjericão & Lamiaceae & 2 & 15,38 \\
\hline Bryophyllum calycinum Salisb. & Pirarucú & Crassulaceae & 2 & 15,38 \\
\hline Gossypium arboreum L. & Algodoeiro & Malvaceae & 1 & 7,69 \\
\hline Portulaca pilosa L. & Amor crescido & Portulacaceae & 1 & 7,69 \\
\hline Aloe vera (L.) Burm. $\mathrm{f}$. & Babosa & Xanthorrhocaceae & 1 & 7,69 \\
\hline Caladium lindenii (André) Madison & Brasileirinho & Araceae & 1 & 7,69 \\
\hline Costus spicatus (Jacq.) Sw. & Canaficha & Zingiberaceae & 1 & 7,69 \\
\hline Cymbopogon citratus (DC.) Stapf & Capim marinho/Capim santo & Poaceae & 1 & 7,69 \\
\hline Aeollanthus suaveolens Mart. ex Spreng. & Catinga-de-mulata & Lamiaceae & 1 & 7,69 \\
\hline Cupressus lusitanica Mill. & Cedrinho & Cupressaceae & 1 & 7,69 \\
\hline Copaifera sp. & Copaibeira & Fabaceae & 1 & 7,69 \\
\hline Pedilanthus tithymaloides (L.) Poit. & Coramina & Euphorbiaceae & 1 & 7,69 \\
\hline Styrax argenteus C. Presl & Estoraque & Styracaceae & 1 & 7,69 \\
\hline Sesamum indicum $\mathrm{L}$. & Gergilim & Pedaliaceae & 1 & 7,69 \\
\hline Mentha pulegium L. & Hortelanzinho & Lamiaceae & 1 & 7,69 \\
\hline Chenopodium ambrosioides L. & Mastruz & Chenopodiaceae & 1 & 7,69 \\
\hline Petiveria alliacea $\mathrm{L}$. & Mucuracaá & Phytolaccaceae & 1 & 7,69 \\
\hline Galinsoga parviflora Cav. & Picão & Asteraceae & 1 & 7,69 \\
\hline Pentaclethra macroloba (Willd.) Kuntze & Pracaxizeiro & Fabaceae & 1 & 7,69 \\
\hline Dalbergia monetaria L. f. & Verônica & Fabaceae & 1 & 7,69 \\
\hline Hibiscus sabdariffa $\mathrm{L}$. & Vinagreira & Malvaceae & 1 & 7,69 \\
\hline
\end{tabular}

\section{DISCUSSÃO}

A faixa etária da população amostrada na comunidade São Tomé foi de 28 a 68 anos. Silva et al. (2013), em trabalho realizado na área do APA do Curiaú, usando metodologia semelhante na escolha dos informantes da aplicada a este trabalho, teve como idade média dos informantes 43 anos, sendo o intervalo compreendido de 20 a 80 anos.

Os dados relacionados ao grau de instrução dos moradores da comunidade são preocupantes, pois a escolaridade é de grande importância para que o homem do campo tenha a capacidade de assimilar as 
inovações tecnológicas, visando melhorar sua produção e a qualidade de vida da família e segundo Quirino et al. (2002) o nível de escolaridade do agricultor na região norte é considerado um dos mais baixos no Brasil e, o ensino fundamental é para $65 \%$ o máximo alcançado para trabalhadores rurais. Sobre a assistência técnica recebida pelos produtores rurais, está é originária do governo estadual, através do órgão de extensão rural (RURAP), que segundo os entrevistados é muito difícil aparecer pela comunidade, não existindo outro tipo de assistência técnica, além da colaboração de agricultores mais experimentados. Quanto ao financiamento para incrementar as atividades produtivas, $70 \%$ dos proprietários declararam que a burocracia é fator que impede o acesso ao crédito, 15\% nunca procurou financiamento, $7 \%$ declararam não ter precisado ou que não aprovam projeto na área. Percebe-se que a burocracia é determinante para acesso ao crédito desses agricultores e que a maioria infelizmente não sabe como conseguir financiamento e nunca foram orientados para essa finalidade.

Com relação aos cuidados dispensados ao quintal, como o plantio de espécies, ou tratos culturais como capina, limpeza de áreas e rega são realizados plenamente por membros familiares da unidade domiciliar e a participação do homem e mulher nesse manejo é efetiva e dominante. Esse resultado deve-se ao fato de que os quintais dos entrevistados possuem grandes extensões, representando grande parte da alimentação e produção do agricultor, necessitando de mão-de-obra masculina e feminina para a manutenção, e também pelo fato dos homens cuidarem das espécies de maior porte e as mulheres de espécies de menor porte, como as plantas medicinais.

De acordo com Silva (2010), na escolha e plantio das espécies vegetais existe uma divisão bem definida, as mulheres possuem um conhecimento mais específico de ervas e arbustos que são cultivados ou nascem espontaneamente nas imediações das unidades domiciliares, enquanto os homens possuem um conhecimento mais especifico das espécies arbóreas, arbustivas e ruderais, que geralmente são encontradas nos caminhos de roças, picadas e matas. Existe a troca de espécies entre vizinhos e parentes e isso faz com que certas espécies tenham ocorrência em vários quintais e, em regra, essas espécies são as de uso medicinal e alimentar.

É importante observar que além dos componentes vegetais, o espaço dos quintais é dividido com as pessoas que compõem as famílias e com a presença de animais domésticos. Em estudo realizado em quintais agroflorestais do Distrito do Carvão, Silva (2010) encontrou a presença de galinhas ( $\sim 7 \%$ dos quintais), patos ( 25\%), porcos ( 12\%) e marrecos ( 8\%). As galinhas são animais criados em oito quintais de São Tomé e em número de indivíduos bem superior aos demais, com um total de 195 unidades, vindo em seguida os patos (33 indivíduos), os porcos (16 indivíduos), os bois (8 indivíduos) e os perus (5 indivíduos). Apenas um entrevistado relatou criar animais (galinhas e patos) para comercializar.

As aves criadas para a produção de carne e ovos são, na maioria dos quintais, criadas soltas, embora exista também a criação em galinheiros preparados especificamente para esse fim. As criações de patos são feitas em três quintais e tem como finalidade produção de carne principalmente para consumo e são criados como as galinhas, tanto de forma solta nos quintais, mas em alguns casos criados confinados. Os porcos são criados unicamente visando à produção de carne, sendo difícil a realização da venda. O confinamento de 
galinhas e patos tem dois objetivos básicos: evitar a fuga dos animais e a proteção contra predadores.

A classificação dos estratos dos QAF em São Tomé apresentou padrão semelhante ao encontrado no trabalho de Silva (2010) em quintais agroflorestais no Distrito do Carvão-AP e estes corroboram com as definições de que os sistemas agroflorestais nos trópicos são caracterizados pela alta diversidade de espécies e distribuídos em quatro estratos verticais.

Os QAF avaliados na comunidade São Tomé apresentaram elevada diversidade de espécies vegetais. Quando se compara esses dados com os encontrados por Almeida et al. (2014) em quintais agroflorestais na comunidade Santo Antônio- PA (60 espécies), Almeida et al. (2014) em quintais de comunidades tradicionais de Barão de Melgaço-MT (82 espécies em Cuiabá Mirim e 96 espécies em Porto Brandão), Miranda et al. (2013) em quintais agroflorestais dos agricultores familiares do Baixo Irituia-PA (125 espécies), Rayol et al. (2013) em quintais agroflorestais desenvolvidos por agricultores familiares do município de Alenquer-PA (33 espécies), Sousa et al. (2011) em quintais do baixo Rio Negro-AM (231 espécies), Sousa et al. (2013) em quintais agroflorestais em comunidades rurais de Santarém-PA (48 espécies), fica evidente que os quintais do São Tomé possuem considerável diversidade vegetal.

Silva (2010), em Distrito do Carvão-AP encontrou a média de 20,90 espécies por quintal, com variação de no mínimo 9 e máximo de 73 espécies. Souza et al. (2013) em estudo da socioeconomia e ecologia de quintais agroflorestais de comunidades rurais de Santarém-PA encontrou a média de 18 espécies por quintal, com variação de no mínimo de 15 e, no máximo 24 espécies. Almeida et al. (2014) pesquisando o uso da biodiversidade em quintais de comunidades tradicionais de Barão de Melgaço-MT encontrou como média 18 espécies e variação de 9 a 38 espécies por quintal em Cuiabá Mirim e média de 19 espécies, com variação de 7 a 33 espécies, em Porto Brandão.

Quanto à riqueza de famílias botânicas identificadas nos QAF, as principais que se destacaram pelo maior número de espécies foram: Rutaceae, Fabaceae e Malvaceae. Almeida et al. (2014) registraram 53 famílias, sendo as mais representativas, pelo número de espécies, Asteraceae, Anacardiaceae, Solanaceae, Annonaceae, Araceae Lamiaceae, Malvaceae, Moraceae, Rutaceae e Verbenaceae. Miranda et al. (2013) estudando quintais agroflorestais dos agricultores familiares do Baixo Irituia-PA, encontrou como famílias mais representativas Musaceae, Rutaceae, Sterculiaceae, Arecaceae, Anacardiaceae, Myrtaceae, Bromeliaceae, Rubiacea e Phasianidae. Rayol et al. (2013) estudando quintais agroflorestais no município de Alenquer-PA encontrou 22 famílias botânicas destacando-se Anacardiaceae, Arecaceae e Myrtceae.

Dentre as espécies que são encontradas de forma espontânea nos quintais, destacaram-se Oenocarpus bacaba (bacabeira), Spondias mombin (taperebazeiro), Caryocar villosum (piquiazeiro), Uncaria tomentosa (unha-de-gato) e Oenocarpus bataua (patauá). As que são encontradas de forma espontânea e também são cultivadas: Euterpe oleracea (açaizeiro), Psidium guajava (goiabeira), Syzygium cumini (ameixeira), Carapa guianensis (andirobeira), Anacardium occidentale (cajueiro), Carica papaya (mamoeiro) e Pouteria caimito (abiuzeiro). Silva (2002) no Curiaú-AP encontrou os valores de $72 \%$ para as cultivadas, 14\% de espécies espontâneas e $15 \%$ de espécies obtidas de forma espontânea e cultivadas. CoelhoFerreira (2009), em Marudá-PA, encontrou 60\% de cultivadas e 35\% de espontâneas. 
As principais categorias de uso das espécies vegetais registradas nos QAF foram a alimentação familiar, uso medicinal e uso madeireiro. Miranda et al. (2013) estudando quintais agroflorestais dos agricultores familiares do Baixo Irituia-PA encontraram como espécies vegetais alimentícias com, porcentagem de frequência relativa de $50 \%$ ou mais de existência nos quintais, a bananeira (Musa spp.), o limoeiro (Citrus limonum), cupuaçuzeiro (Theobroma grandiflorum), pupunheira (Bactris gasipaes), cajueiro (Anacardium orcidentale), goiabeira (Psidium Guayaba), mangueira (Mangifera indica), laranjeira (Citrus sinensis), cafeeiro (Coffea arabica), abacaxizeiro (Ananas comosus) e açaizeiro (Euterpe oleracea).

Lacerda et al. (2011) pesquisando espécies vegetais nos quintais agroflorestais da comunidade Cabeça Branca-PB, registrou como espécies mais frequentes: Annona squamosa L., Carica papaya L., Cocos nucifera L., Malpighia glabra L., Psidium guajava L., Anacardium occidentale L., Annona muricata L. e Lippia alba (Mill.) Brow., Caesalpinia pyramidalis Tul., Citrus sinensis (L.) Osbeck, Cymbopogon citratus Stapf, Mangifera indica L. e Spondias purpurea L. Vilarinho et al. (2011) estudando quintais agroflorestais no Município de Salvaterra-Marajó-PA encontraram como espécies mais frequente o coqueiro, a bananeira, limoeiro e mamoeiro. Sousa et al. (2013) analisando quintais agroflorestais em comunidades rurais de Santarém-PA registraram como espécies mais frequentes a laranjeira, abacateiro, goiabeira, cupuaçuzeiro, coqueiro, mangueira, limoeiro, bananeira, aceroleira e ingazeiro.

O aspecto alimentício geralmente é considerado como o principal motivo para a implantação dos quintais agroflorestais. Esse resultado é semelhante aos encontrados por Miranda et al. (2011), Miranda et al. (2013). Assim, os quintais agroflorestais garantem a oferta e a diversidade de alimentos para suprir as necessidades nutricionais dos membros das unidades familiares de produção. Dessa forma, os cocos, as mangas, os cupus, banana e pupunha são os frutos mais consumidos e fazem parte da paisagem local, na composição dos quintais e são consumidos, principalmente pelas crianças. Mas vale ressaltar que esses produtos constituem em uma fonte de renda, pois são vendidos geralmente in natura na feira do município de Ferreira Gomes-AP.

As palmeiras apresentam importância fundamental na alimentação dos moradores, como a Euterpe oleracea Mart. (açaizeiro), Cocos nucifera L. (coqueiro), Bactris gasipaes Kunth (pupunheira) e a Oenocarpus bacaba Mart. (bacabeira), que são encontrados espontâneos nos quintais e nas proximidades, com alguns espécimes cultivados. Dezesseis espécies apresentam um percentual de ocorrência nos QAF entre 30\% a $46 \%$ e 29 das espécies possuem percentual abaixo de $25 \%$, sendo que 15 destas aparecem somente em um quintal.

As espécies medicinais mais representativas por ocorrência nos QAF foram: Carapa guianensis (andirobeira), Carica papaya (mamoeiro), Vernonia condensata Baker (boldo), Lippia alba (erva cidreira/cidreira) e as espécies Ruta graveolens (arruda), Piper callosum (elixir paregórico), Plectranthus amboinicus (hortelã), Morinda citrifolia (noni), Arrabidaea chica (pariri), Uncaria tomentosa (unha-de-gato). Esses dados só reforçam a potencialidade existente nos quintais no que tange as plantas medicinais, alimentícias, bem como o conhecimento que a comunidade é detentora sobre esses recursos. Deve-se destacar que neste levantamento não foram incluídas as espécies medicinais que são encontradas fora dos 
quintais agroflorestais, como por exemplo na floresta nativa.

\section{CONCLUSÕES}

Os QAF apresentaram alta diversificação de espécies, sendo sua composição florística e distribuição determinadas por fatores externos e internos, como função e tamanho do quintal, bem como fatores socioeconômicos e culturais, além da influência direta da família que seleciona as espécies de acordo com suas necessidades.

Das 58 espécies utilizadas pela comunidade na alimentação ou complemento desta, as que tiveram maior destaque foram: Cocos nucifera, Mangifera indica, Theobroma grandiflorum, Musa cavendishii, Bactris gasipaes, Euterpe oleracea, Psidium guajava, Citrus limonia, Syzygium cumini, Citrus sinensis, Annona muricata, Inga edulis, Citrus nobilis, todas com mais de 50\% de frequência nos quintais.

Foram registradas nos QAF 31 espécies utilizadas como plantas medicinais na comunidade, sendo que $~ 70 \%$ dos entrevistados faziam uso destas. As espécies mais representativas por ocorrência foram: Carapa guianensis, Carica papaya, Vernonia condensata, Lippia alba, Ruta graveolens, Piper callosum, Plectranthus amboinicus (Lour.) Spreng., Morinda citrifolia, Arrabidaea chica, Uncaria tomentosa. O conhecimento sobre plantas medicinais vem de tradição familiar e o grau de estudo não é fundamental no conhecimento e uso das plantas medicinais e alimentícias.

O manejo dos QAF é simples e de baixo custo, uma vez que envolve práticas tradicionais de cultivo de plantas e a principal finalidade dos quintais na comunidade São Tomé é de promover a complementação alimentar das unidades familiares, e de venda de sua produção na feira. Desta forma, pode-se afirmar que os quintais agroflorestais da comunidade São Tomé são um sistema de produção de autoconsumo familiar e renda, contribuindo para a melhoria da alimentação da população local, ajudam a melhorar a capacidade produtiva da terra e contribuem para a proteção do meio ambiente.

\section{REFERÊNCIAS}

ALMEIDA, L. S.; GAMA, J. R. V.. Quintais agroflorestais: estrutura, composição florística e aspectos socioambientais em área de assentamento rural na Amazônia brasileira. Ciência Florestal, Santa Maria, v.24, n.4, p.1041-1053, 2014.

ALMEIDA, S. E.; PASA, M. C.; GUARIM, V. L. M. S.. Uso da biodiversidade em quintais de comunidades tradicionais da Baía de Chacorore, Barão de Melgaço, Mato Grosso, Brasil. Biodiversidade, v.13, n.1, p.141-155, 2014.

ALVES, F. V.; LAURA, V. A.; ALMEIDA, R. G.. Sistemas agroflorestais: a agropecuária sustentável. Brasília: Embrapa, 2015

AMAPÁ. Secretaria Especial de Desenvolvimento Econômico e Secretaria de Estado do Meio Ambiente, Governo do Estado do Amapá. Plano de prevenção e controle do desmatamento e queimadas do estado do Amapá: Contexto e Ações. Macapá: PPCDAP, 2009.

AMOROZO, M. C. M.. A abordagem etnobotânica na pesquisa de plantas medicinais. In: DISTASI, L. C.. Plantas medicinais: arte e ciência: um guia de estudo interdisciplinar. Botucatu: UNESP, 1996. p.47-68.

APG III. Angiosperm Phylogeny Group. The Linnean Society of London. Botanical journal of the Linnean Society. APG, 2009.

CARNEIRO, M. G. R.; MACHADO, A. C.; ESMERALDO, G. G. S. L.; SOUSA, N. R.. Quintais produtivos: contribuição à segurança alimentar e ao desenvolvimento sustentável local na perspectiva da agricultura familiar ( $O$ caso do assentamento Alegre, município de Quixeramobim/CE). Revista Brasileira de Agroecologia, Porto Alegre, v.8, n.2, p.135-147, 2013.

CARVALHO, A. J. A.; SOUZA, E. H.; MARQUES, C. T. S.; GAMA, E. V. S.; NACIF, P. G. S.. Caracterização física dos solos dos quintais agroflorestais e cultivos monotípicos na região de Amargosa, Bahia. Revista Brasileira de Agroecologia, v.2, p.941-944, 2007.

COELHO-FERREIRA, M.. Medicinal knowledge and plant 
utilization in an Amazonian coastal community of Marudá, Pará State (Brazil). Journal of Ethnopharmacology, v.126, n.1, p.159-175, 2009. DOI:

https://doi.org/10.1016/j.jep.2009.07.016

DAS, T.; DAS, A. K.. Inventorying plant biodiversity in homegardens: a case study in Barak Valley, Assam, north east Índia. Current Science, Bangalore, v.89, n.1, p.155-163, 2005.

ECOTUMUCUMAQUE. Estudo de Impacto Ambiental do Aproveitamento Hidrelétrico Ferreira Gomes. Macapá, 2010.

FERREIRA GOMES. Plano diretor participativo do município de Ferreira Gomes, Estado do Amapá. Ferreira Gomes, 2013.

FREITAS, A. V. L.; COELHO, M. F. B.; PEREIRA, Y. B.; FREITAS NETO, E. C.; AZEVEDO, R. A. B.. Diversidade e usos de plantas medicinais nos quintais da comunidade de São João da Várzea em Mossoró, RN. Revista Brasileira Plantas Medicinais, Botucatu, v.17, n.4, p.845-856, 2015.

GIL, A. C.. Métodos e Técnicas de Pesquisa Social. 5 ed. São Paulo: Atlas, 1999.

GOMES, K. B. P.; MARTINS, R. C. C.; DIAS, C. A.; MATOS, J. M. M.. Quintais agroflorestais: características agrossociais sob a ótica da agricultura familiar. Revista Ibero Americana de Ciências Ambientais, v.9, n.4, p.111-124, 2018. DOI: http://doi.org/10.6008/CBPC2179-6858.2018.004.0009

LACERDA, A. V.; BARBOSA, F. M.; GOMES, A. C.; SILVA, D. V.; ALCÂNTARA, H. M.; ISIDRO, R.. Análise da distribuição das espécies vegetais nos quintais agroflorestais da comunidade Cabeça Branca, região do Cariri paraibano, Brasil. Cadernos de Agroecologia, Fortaleza, v.6, n.2, 2011.

MARTINS, E. M.; SILVA, E. R.; CARNEIRO, E. F.; CAMPELLO, S. S. L.; NOBRE, C. P.; CORREIA, M. E. F. C.; RESENDE, A. S.. O uso de sistemas agroflorestais diversificados na restauração florestal na Mata Atlântica. Ciência Florestal, Santa Maria, v.29, n.2, p.632-648, 2019. DOI: https://doi.org/10.5902/1980509829050

MIRANDA, S. B.; KATO, O. R.; SABLAYROLLES, M. G. P.. Quintais agroflorestais e segurança alimentar de agricultores familiares no Baixo Irituia, Nordeste paraense. EMBRAPA, 2011.

MIRANDA, S.; KATO, O.; SABLAYROLLES, M. G.

Caracterização e importância dos quintais agroflorestais aos agricultores familiares do Baixo Irituia, Pará. Cadernos de Agroecologia, Porto Alegre, v.8, n.2, 2013.
NAIR, P. K. R.. Agroforestry Systems and Environmental Quality: Introduction. Journal of Environmental Quality, v.40, n.3, p.784-90, 2011.

PEYRE, A. G.; WIERSUM, K. F.; BONGERS, F.. Dynamics of homegarden structure and function in Kerala, India. Agroforestry Systems, Chennai, v.66, n.2, p.101-115, 2006. DOI: https://doi.org/10.1007/s10457-005-2919-x

QUIRINO, T. R.; GARAGORRY, F. L.; SOUSA, C. P.. Diagnóstico sociotécnico da agropecuária brasileira: I Produtores. Brasília: Embrapa Informação Tecnológica, 2002.

RAYOL, B. P.; ALVINO-RAYOL, F. O.; SILVA, M. C. P.. Caracterização de quintais agroflorestais desenvolvidos por agricultores familiares do município de Alenquer, Pará. Cadernos de Agroecologia, Porto Alegre, v.8, n.2, 2013.

RAYOL, B. P.; MIRANDA, I. S.. Quintais agroflorestais na Amazônia Central: caracterização, importância social e agrobiodiversidade. Ciência Florestal, Santa Maria, v.29, n.4, p.1614-1629, 2019. DOI:

https://doi.org/10.5902/1980509829853

SILVA, R. B. L.. A etnobotânica de plantas medicinais da comunidade quilombola de Curiaú, Macapá-AP, Brasil. Dissertação (Mestrado em Agronomia) - Faculdade de Ciências Agrárias do Pará, Belém, 2002.

SILVA, R. B. L.. Diversidade, uso e manejo de quintais agroflorestais no Distrito do Carvão, Mazagão-AP, Brasil. Tese (Doutorado em Desenvolvimento Sustentável do Trópico Úmido) - Universidade Federal do Pará, Belém, 2010.

SILVA, R. B. L.; FREITAS, J. L.; SANTOS, J. U. M.; SOUTO, R. N P.. Caracterização agroecológica e socioeconômica dos moradores da comunidade quilombola do Curiaú, MacapáAP, Brasil. Biota Amazônia, Macapá, v.3, n.3, p.113-138, 2013.

SOUSA, C. C. V.; SCUDELLER, V. V.. Diversidade vegetal nos quintais do baixo Rio Negro, Manaus (AM). Cadernos de Agroecologia, Fortaleza, v.6, n.2, 2011.

SOUSA, W. A.; VIEIRA, T. A.; LUSTOSA, D. C.. Socioeconomia e ecologia de quintais agroflorestais em comunidades rurais de Santarém, Pará. Cadernos de Agroecologia, Porto Alegre, v.8, n.2, 2013.

VILARINHO, C.; BARBOSA, C.; NAZARÉ, N.; SILVA, J.; PINTO, W.. Quintais agroflorestais (QAF) no Município de Salvaterra-Marajó e suas contribuições para o desenvolvimento socioeconômico do município. Cadernos de Agroecologia, Fortaleza, v.6, n.2, 2011.

A CBPC - Companhia Brasileira de Produção Científica (CNPJ: 11.221.422/0001-03) detém os direitos materiais desta publicação. Os direitos referem-se à publicação do trabalho em qualquer parte do mundo, incluindo os direitos às renovações, expansões e disseminações da contribuição, bem como outros direitos subsidiários. Todos os trabalhos publicados eletronicamente poderão posteriormente ser publicados em coletâneas impressas sob coordenação da Sustenere Publishing, da Companhia Brasileira de Produção Científica e seus parceiros autorizados. Os (as) autores (as) preservam os direitos autorais, mas não têm permissão para a publicação da contribuição em outro meio, impresso ou digital, em português ou em tradução. 